\title{
Visual Inspection of Geological Maps: an eye-tracking Study
}

\author{
Stanislav Popelka ${ }^{\mathrm{a}, *}$, Jiř́i Komínek ${ }^{\mathrm{a}}$ \\ ${ }^{a}$ Department of Geoinformatics, Palacký University Olomouc, Czech Republic, Stanislav Popelka - stanislav.popelka@upol.cz, Jiři \\ Kominek-kominekjiri@seznam.cz \\ * Corresponding author
}

Keywords: cartography, geology, eye-tracking, evaluation, user study

\begin{abstract}
:
The paper describes the analysis of a visual inspection of paper geological maps by three groups of participants: geologists (GEOL), geographers (GEO) and geoinformaticians (GIS). The aim of the study was to identify the differences in how different groups of participants visually inspected geological maps.

Geological maps show the distribution of different types of rock at the earth's surface and are a fundamental tool for geologists. Geology as a distinct discipline is relatively young, and its origins date to the eighteenth century. In recent years, this otherwise relatively stable field has begun to incorporate new technology into its tools and methods. No study as yet has been published to assess geological maps using eye-tracking or cognitive cartography.
\end{abstract}

Eye-tracking glasses SMI Eye Tracking Glasses 2 with a recording frequency of $60 \mathrm{~Hz}$ were used to record the eyemovements of participants during the experiment. Two maps at a scale of $1: 25000$ produced by the Czech Geological Survey were used for the eye-tracking experiment. In the first part of the experiment, free viewing was analyzed. In the second part, participants solved six tasks with a map. The tasks were selected based on consultation with employees of the Czech Geological Survey.

In the free viewing section, noticeable differences between groups were observed. The free viewing section revealed that the geoinformaticians group concentrated much less on the map itself and spent more time on the surrounding elements. The geographers and geologists mainly focused on the map field. The second part of the experiment comprised six tasks.

The first task was the simplest and involved finding the coordinate system used in the map. The task caused no problems in any group, and completion times were balanced. The GIS group demonstrated the greatest experience in reading maps and was also the quickest to solve the task. The second task was to identify the geological units depicted in the map. The correct answer could be obtained either from the legend or by using the scheme of geological units. No statistically significant differences in time between the groups were recorded. In the third task, respondents were required to identify and mark the boundary between two geological units found in the previous task. The group of geologists was quickest to solve this task, and all other respondents found a solution with no great difficulty. The fourth task was to identify the predominant rock and determine its type according to the legend. The results suggest that ten respondents from GEO and GIS groups who concentrated on the lithostratigraphic scheme did not know where to look for the correct answer. In the fifth task, participants were required to mark an area with multiple landslides. The aim in this task was to find the landslide symbol in the legend and then identify the landslide area on the map. The differences between groups were most apparent in this task. The geologists were significantly quicker in finding the symbol in the legend. The other groups needed a much longer time to identify the symbol in the legend. In the final task, respondents were instructed to identify the predominant rock in the area with the highest amplitude of geomagnetic anomalies. To solve this task, using the diagram in the section at the bottom left was necessary. The GEOL group spent the least amount of time completing this task, indicating the respondents' knowledge. The experiment and subsequent interviews revealed a different color reading strategy. When identifying a rock, the GEOL group compared colors mainly for quick orientation. However, the decisive factor for identification was the index, which was given for the rock in the map and legend.

To conclude, the geologists group was quickest in solving the tasks and recorded the least wrong answers. The GIS and GEO groups achieved similar results in the experiment.

\section{Acknowledgements}

The research was supported by the Czech Science Foundation, project no. 18-05432S. 